\title{
Fano resonance boosted cascaded optical field enhancement in a plasmonic nanoparticle-in-cavity nanoantenna array and its SERS application
}

\begin{abstract}
Zhendong Zhu ${ }^{1,2}$, Benfeng Bai ${ }^{1}$, Oubo You ${ }^{1}$, Qunqing $\mathrm{Li}^{2}$ and Shoushan Fan ${ }^{2}$
Cascaded optical field enhancement (CFE) can be realized in some specially designed multiscale plasmonic nanostructures, in which the generation of extremely strong fields at nanoscale volume is crucial for many applications, for example, surface-enhanced Raman spectroscopy (SERS). In this paper, we propose a strategy for realizing a high-quality plasmonic nanoparticle-in-cavity (PIC) nanoantenna array, in which strong coupling between a nanoparticle (NP) dark mode with a high-order nanocavity bright mode can produce strong Fano resonance at the target wavelength. The Fano resonance can effectively boost the CFE in a PIC. A cost-effective and reliable nanofabrication method is developed using room temperature nanoimprinting lithography to manufacture high-quality PIC arrays. This technique guarantees the generation of only one gold NP at the bottom of each nanocavity, which is crucial for the generation of the expected CFE. To demonstrate the performance and application of the PIC array, the PIC array is employed as an active SERS substrate for detecting 4-aminothiophenol molecules. An experimental SERS enhancement factor of $2 \times 10^{7}$ is obtained, which verifies the field enhancement and the potential of this device.
\end{abstract}

Light: Science \& Applications (2015) 4, e296; doi:10.1038/lsa.2015.69; published online 05 June 2015

Keywords: cascaded optical field enhancement; Fano resonance; localized surface plasmon; plasmonic nanoantenna; surfaceenhanced Raman spectroscopy

\section{INTRODUCTION}

Metallic nanostructures that support surface plasmons (SPs) have attracted significant attention due to their peculiar optical properties and important applications. ${ }^{1,2}$ SPs can be considered to be the resonant photon-induced collective oscillations of free electrons confined on the surfaces of metallic nanostructures, which can be driven by an incident light field. The importance of these photonelectron interactions is their ability to concentrate light energy in nanoscale volumes in the metallic nanostructures and subsequently boost the intensity of the optical near field by several orders of magnitude. $^{2-5}$ Due to these properties, localized SPs can facilitate many applications based on the enhanced light-matter interaction, such as the detection of trace element chemical varieties in the vicinity of metallic nanostructures by surface-enhanced Raman spectroscopy (SERS). The detection limit can be reduced to a single molecular level. $^{6,7}$

The SP-enhanced optical near field (also referred to as the field 'hot spot $^{6,7}$ ) is typically and predominantly localized in very small geometric features of metallic nanostructures, such as nano-grooves, ${ }^{2}$ nanogaps, ${ }^{8}$ and nanotips. ${ }^{9}$ Although certain advances have been achieved regarding the fabrication of nanostructures with extremely small fea ture sizes, ${ }^{10}$ these tiny features remain formidable tasks for nanofabrication. Alternatively, extreme field localization and enhancement via a cascaded optical field enhancement (CFE) mechanism may be realized. This concept was proposed by Stockman et al. with a self-similar chain of gold nanoparticles (NPs), ${ }^{2,3}$ in which the light energy can be focused to a nanoscale volume via the intercoupling of localized SPs among the aligned NPs with decreasing sizes. Field enhancement is achieved by a relatively large metallic NP that is resonantly excited by the incident light. Then, the enhanced field simultaneously excites the field of the adjacent smaller NP via SP coupling. The process continues until the smallest NP is excited and the light energy is predominantly confined in the tiniest nanogap. ${ }^{3}$ Although self-similar chains of NPs have shown great potential, fabrication of these nanofocusing systems in practice is challenging. The cascaded energy focusing effect exists not only in the self-similar chains of metal $\mathrm{NPs}^{3}$ but also in many other metallic nanostructures with multiscale features, such as V-grooves, ${ }^{2}$ nanostars, ${ }^{8}$ ellipsoidal NP dimers, ${ }^{11}$ and asymmetric $\pi$-shaped metamaterials. ${ }^{12}$ Few experimental verifications of the CFE effect in certain geometries have been reported. ${ }^{13}$ Recently, Kravets et al. noted CFE in a composite plasmonic system, in which each unit is composed of two or three nanodisks. ${ }^{14,15}$ Novotny et al. demonstrated CFE in arrays of gold NPs that were assembled into dimmer or trimer nanoantennas. ${ }^{16}$ These novel CFE plasmonic nanostructures show great potential for applications such as SERS with a single molecular level, ${ }^{7}$ high-sensitivity reflective index sensing, ${ }^{17}$ and super-resolution imaging. ${ }^{9,18}$ In our previous

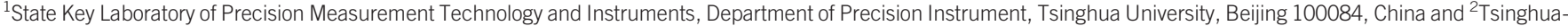
Foxconn Nanotechnology Research Center, Department of Physics, Tsinghua University, Beijing 100084, China.

Correspondence: Dr Benfeng Bai, Department of Precision Instrument, Tsinghua University, Beijing 100084, China

E-mail: baibenfeng@mail.tsinghua.edu.cn

Received 10 November 2014; revised 10 February 2015; accepted 3 March 2015; accepted article preview online 7 March 2015 
study of M-shaped nanograting, strong CFE can be achieved by the intercoupling between the localized SPs in two adjacent V-grooves, which produces pronounced enhancement of the SERS sensitivity. ${ }^{19}$

Another effective method of tuning the resonance in a complex plasmonic system is mode hybridization, which results from the interference between two or more plasmonic modes. ${ }^{1,5}$ A plasmonic mode can be either subradiant (referred to as the dark mode) or superradiant (referred to as the bright mode), depending on how strong the incident light can be coupled to the plasmonic mode. ${ }^{20,21}$ Analogous to a classical Fano resonance system, ${ }^{1,5,22}$ plasmonic Fano resonance may also occur from the interference between a continuum of incident photons or a spectrally broad resonance (e.g., caused by a super-radiant bright mode) with a narrow resonance (e.g., caused by a subradiant dark mode). The intercoupling between the bright mode and the dark mode occurs when the near field of one mode overlaps with and excites the near field of the other mode, which can be generated in certain coupled plasmonic nanostructures, such as the symmetry breaking nanocavity-NP system, ${ }^{21}$ the nonconcentric ring-disk cavity, ${ }^{23}$ the plasmonic nanoshell, ${ }^{24} \mathrm{NP}$ oligomer chains, ${ }^{25}$ nanovoids, ${ }^{26-30}$ nanostars, ${ }^{31}$ and nanogratings. ${ }^{32}$ The plasmonic Fano interference usually results in an asymmetric resonant peak dressing within a broad spectrum. ${ }^{33}$

Complex plasmonic nanostructures, in which individual tiny metal NPs are coupled to certain extended metallic structures, are ideal systems for generating both CFE and mode hybridization, such as the plasmonic nanoparticle-in-cavity (PIC) nanoantenna array. ${ }^{26-30}$ Cole et al. have systematically analyzed the excited fields and energies of various localized modes in empty truncated plasmonic nanocavities embedded in a metal film, in which various bonding and anti-bonding hybridized modes can be generated. ${ }^{26-29}$ Huang et al. have theoretically analyzed a PIC structure and revealed that the CFE can be effectively induced by the intercoupling between the ${ }^{0} \mathrm{D}$ mode of the empty void and a 'NP mode' induced by the gold NP located at the bottom of the cavity under oblique incidence. ${ }^{30}$ To verify the CFE effect, they fabricated PIC structures (with $20 \mathrm{~nm} \mathrm{Ag} \mathrm{NPs} \mathrm{in} 600 \mathrm{~nm}$ Au voids) and employed them as SERS substrates to probe 4-aminothiophenol (4-ATP) molecules, from which a SERS enhancement factor of $3.1 \times$ $10^{4}$ was achieved. ${ }^{30}$ This SERS enhancement factor is relatively low because the assembly method cannot precisely control the number and allocation of Ag NPs in the Au voids. Approximately 10\% Au voids surface was coated with randomly distributed Ag NPs after the bare Au void substrate was immersed in a $20 \mathrm{~nm}$ Ag NPs solution. Even within the coated area, some voids may contain no NP or may contain several NPs (which may even aggregate to form larger NPs). Therefore, the expected CFE effect cannot be readily and efficiently realized. In addition, the coupling between the ${ }^{0} \mathrm{D}$ mode and the NP mode in the PIC for generating the CFE requires adequate control of an oblique incident angle of approximately $60^{\circ}$, which is crucial for applications. Efforts were also made by other researchers regarding the fabrication of PICs by other methods, such as nanosphere lithography assisted by an oblique deposition technique and annealing treatment. ${ }^{26-30,34-37}$ However, obtaining a single gold NP dressing in each nanovoid is difficult and the PIC array can only be arranged in closely packed hexagonal lattice. The randomness of the NP allocation in these fabricated PIC structures is a significant problem that substantially degrades the expected CFE effect.

By summarizing the previous studies on PICs and attempting to achieve their practical SERS applications, we determine the need to solve the following main issues: first, a relatively simple PIC design is required for generating strong CFE for a SERS excitation wavelength, preferably under normal incidence rather than the oblique incidence at a fixed angle. ${ }^{30}$ Second, small PICs with void diameters less than $200 \mathrm{~nm}$ and a NP size less than $30 \mathrm{~nm}$ are important for generating Fano resonance and CFE in visible to near-infrared spectra to fit SERS excitation wavelengths, which have been less examined. Third, a costeffective and robust fabrication method is necessary to fabricate highquality PICs with a strong CFE effect. Only one NP should be located at the bottom of each void because the slight change in the location, aggregation, and number of NPs in the void may significantly influence the mode hybridization and the induced CFE effect. ${ }^{30}$

In this study, we propose a strategy for realizing a high-quality plasmonic PIC nanoantenna array by considering the three previously mentioned main issues. Our objective is to achieve a strong CFE effect boosted by Fano resonance with normal incidence at a given wavelength. A cost-effective, efficient, and reliable nanofabrication technique that involves room temperature nanoimprinting lithography (RTNIL) ${ }^{19}$ and anisotropic reactive ion etching (RIE) is developed to manufacture the PICs. Because the PICs can potentially produce extremely strong CFE, which may be even stronger than the CFE produced by other plasmonic oligomers (such as dimers, ${ }^{38}$ trimers, ${ }^{39}$ and nanofingers ${ }^{40}$ ), they can be potentially exploited for single molecular sensing. To analyze the sensing performance of the PIC array, we employ the PIC arrays as SERS substrates for probing 4-ATP molecules; the SERS enhancement factor is experimentally evaluated.

\section{MATERIALS AND METHODS}

The geometry of the PIC nanoantenna unit is depicted in Figure 1a. A gold NP with the diameter $d_{\mathrm{p}}$ is located at the bottom of a truncated gold cavity with the diameter $d_{\text {c }}$. The cavity is formed by etching a gold nanocube with side lengths of $l$ and a height (which is also the height of the truncated nanocavity) of $t$. The nanoantenna array is situated on a fused silica substrate. The lattice constants along the $x$ and $z$ directions are $p$. The PIC array is assumed to be illuminated by an $x$-polarized plane wave at normal incidence from the top side. It is designed to produce strong CFE at an expected SERS excitation wavelength of $720 \mathrm{~nm}$.

The optical response of the structure is numerically simulated with a commercial full-wave software CST Microwave Studio based on the finite integral method. ${ }^{41}$ A $3 \mathrm{~nm}$ thick $\mathrm{Cr}$ adhesion layer is situated between the gold layer and the quartz substrate. The permittivity function of $\mathrm{Au}$ and $\mathrm{Cr}$ are obtained from the experimental data and the Palik database. ${ }^{42}$ The refractive index of the fused silica substrate is assumed to be 1.5 by neglecting its small dispersion. To understand the physics and unique properties of the PIC array, we compare it with a truncated empty nanocavity (ENC) array in the following analyses. The structural parameters of the ENC array are equivalent to the structural parameters of the PIC array; the only difference is that there is no NP in each nanocavity of the ENC array.

We have developed a reliable technique involving RT-NIL and anisotropic RIE to fabricate the PIC array, which is one of the main contributions of this study. Figure 2a describes the nanofabrication process. Two layers of photoresists, including a hydrogen silsesquioxane (HSQ, Fox-14, Dow Corning Inc.) top layer with a thickness of $150 \mathrm{~nm}$ and a polymethylmethacrylate-co-polystyrene copolymer (PMMA-b-PS, which is a homemade imprinting resist with PMMA/ PS: $21000 / 64000 \mathrm{~g} \cdot \mathrm{mol}^{-1}$ ) bottom layer with a thickness of $250 \mathrm{~nm}$, were spin-coated onto a $60 \mathrm{~nm}$ thick gold film coated on a fused silica substrate. After prebaking, the RT-NIL process was operated with a pressure of $50 \mathrm{psi}$ at room temperature. The silicon stamp consists of a crossed grating with cubic profiles fabricated by standard electronbeam lithography; the lattice constants along the $x$ and $z$ directions are 

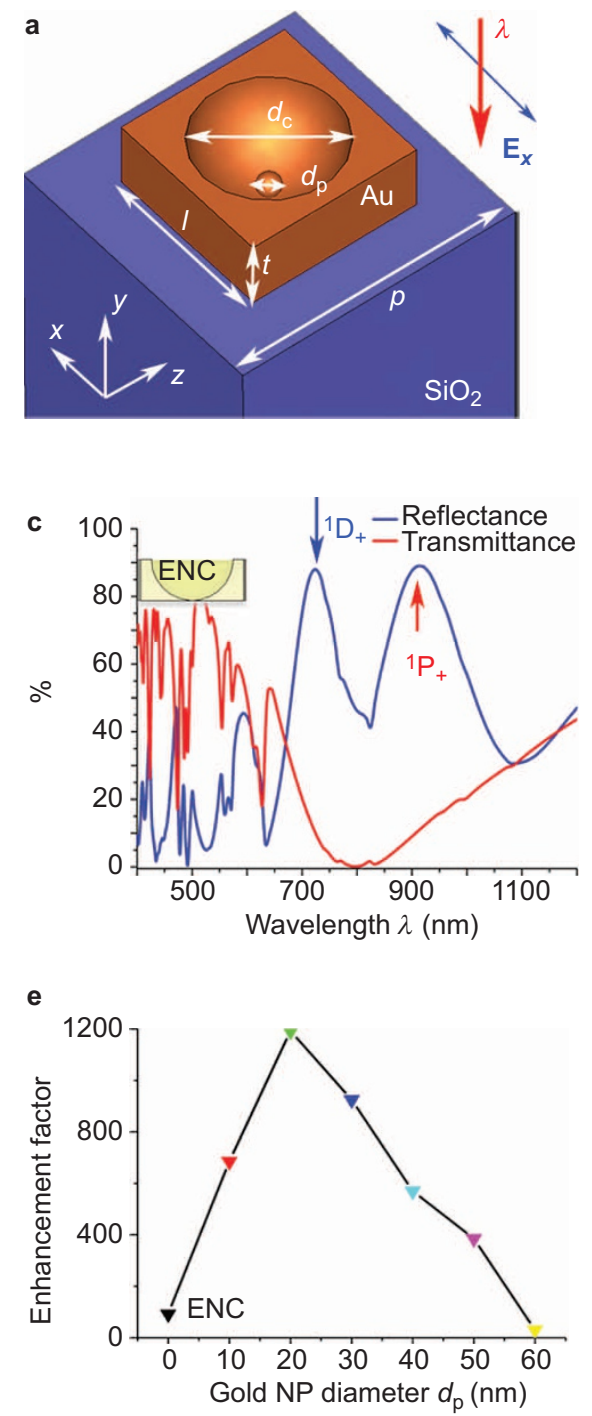
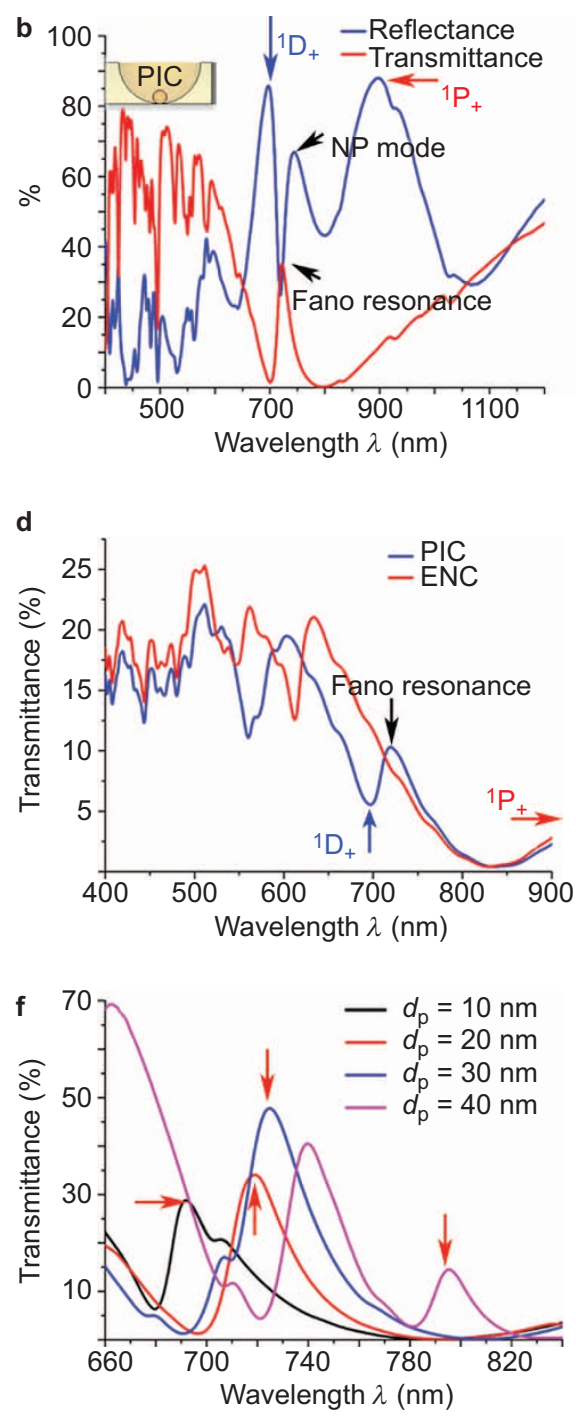

Figure 1 (a) Schematic geometry of the unit cell of a two-dimensionally periodic PIC array, which is illuminated by an $x$-polarized plane wave under normal incidence. (b) and (c) are the numerically simulated far-field transmittance and reflectance spectra of the PIC array and the reference ENC array, respectively. The resonance peaks of the ${ }^{1} D_{+}$and ${ }^{1} P_{+}$cavity modes, the NP mode, and the Fano resonance are indicated by arrows. (d) The experimentally measured transmittance spectra of a fabricated PIC array and an ENC array. (e) The calculated field amplitude enhancement factor in the PIC array with respect to an increase in the gold NP diameter. (f) The simulated transmittance spectra of the PIC array with different gold NP sizes, where the Fano resonance positions are indicated by arrows.

$250 \mathrm{~nm}$, and the width and depth of the cubic unit are $150 \mathrm{~nm}$ and $250 \mathrm{~nm}$, respectively. After releasing, the HSQ in the imprinted grooves was removed by a RIE process with pure $\mathrm{CF}_{4}(40 \mathrm{sccm}$, $2 \mathrm{~Pa}, 40 \mathrm{~W}, 10 \mathrm{sec}$ ). Then, another $\mathrm{O}_{2} / \mathrm{CF}_{4}$ composite RIE process $(20 \mathrm{sccm} / 48 \mathrm{sccm}, 2 \mathrm{~Pa}, 40 \mathrm{~W}, 90 \mathrm{sec})$ was operated to etch the residual PMMA in the grooves and simultaneously etch the top HSQ layer.

Prior to the subsequent procedures, an important step of thermal annealing is necessary to induce the phase separation in the PMMA-bPS photoresist. The sample was rapidly heated to $175^{\circ} \mathrm{C}$ in one minute, maintained at this temperature for $5-7 \mathrm{~min}$, and rapidly cooled to $25^{\circ} \mathrm{C}$ within 10-30 sec. The PMMA-b-PS layer received an intersurface tension between PMMA and PS during the annealing, ${ }^{43}$ which caused PMMA to diffuse outward while PS remained relatively stable. Consequently, the PMMA-b-PS layer became a gradient resist with varying etching resistance from the center to the outer area. The induced tensile stress and diffusion of PMMA cause the collapse of the resist in the central area of the cubic resist block and the formation of a resist cavity with an aggregated resist particle at the bottom. Then, this anisotropic bilayer resist with a special geometry serves as the mask for etching the gold film located underneath.

With the HSQ and PMMA-b-PS bilayer mask, an anisotropic RIE process was employed with $\mathrm{O}_{2} 5 \mathrm{sccm} / \mathrm{Ar} 20 \mathrm{sccm} / \mathrm{Cl}_{2} 10 \mathrm{sccm}$ plasma. During this process, the bowl-shaped cavity was transferred to the gold layer by dry etching ( $70 \mathrm{~W}, 16 \mathrm{~Pa}, 120 \mathrm{sec})$; because the central small resist particle in the cavity is thicker than the adjacent resist, the gold underneath this resist particle was etched more slowly than the gold in the adjacent area. In this manner, the gold NP in the cavity was gradually formed with an appropriately selected etching time and recipe. For details on the fabrication process (including the dry etching of the gold film, the preparation of the bilayer resist mask, the formation of the gold NP during RIE, the control of the gold NP size and position, and the stability and purity of the fabricated gold PIC array), readers may refer to Section 1 of the Supplementary Information. 


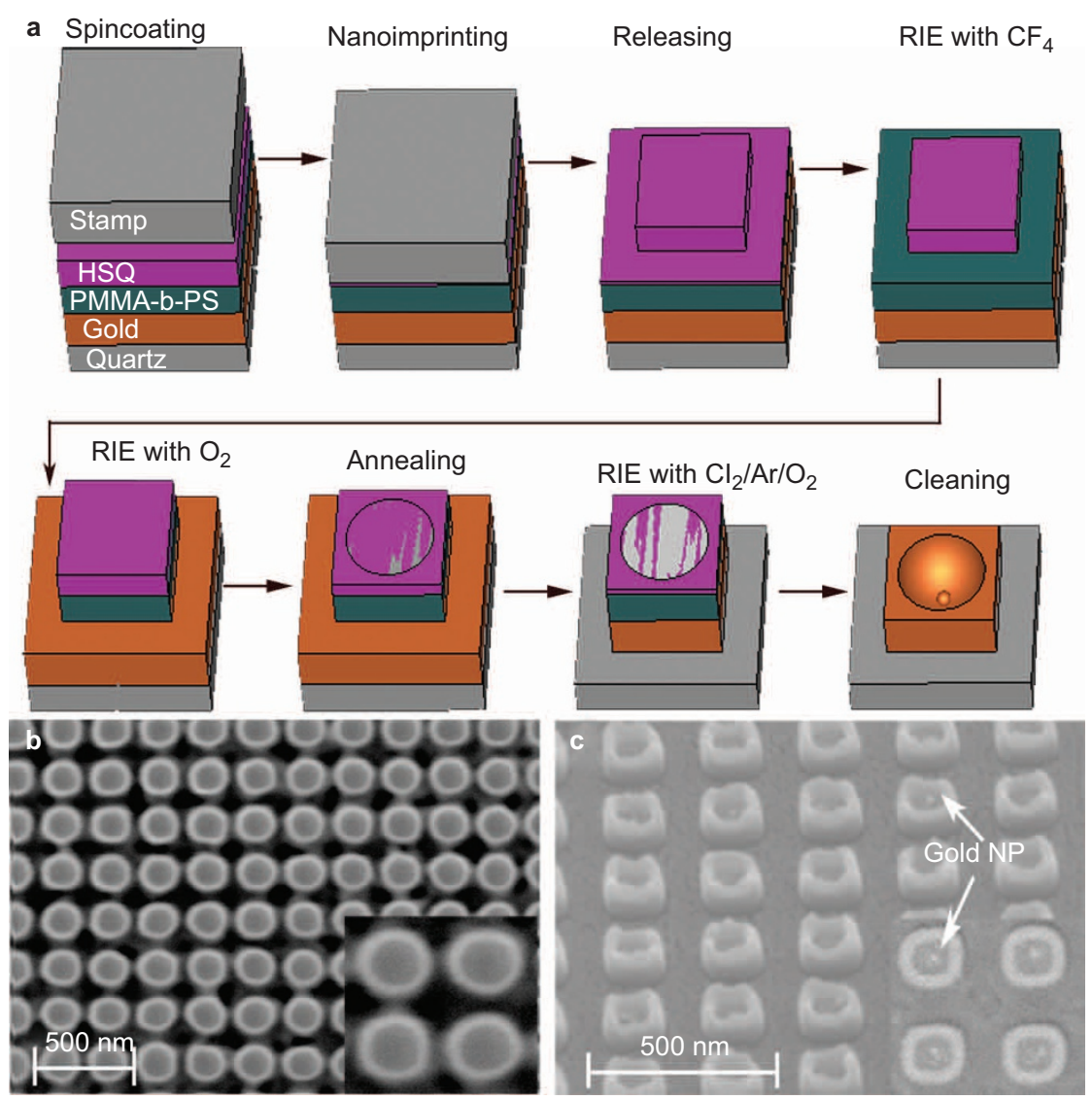

Figure 2 (a) Schematic of the fabrication process of the PIC array using a technique based on RT-NIL and anisotropic RIE. (b) SEM image of a fabricated ENC array, in which the inset shows an enlarged image of the sample. (c) SEM image of a fabricated PIC array, in which the inset shows an enlarged top-view image of the sample.

Note that the size of the gold NPs can be controlled by tuning the anisotropic RIE parameters. For example, with a RIE recipe of $\mathrm{O}_{2}$ $10 \mathrm{sccm} / \mathrm{Ar} 20 \mathrm{sccm} / \mathrm{Cl}_{2} 10 \mathrm{sccm}, 16 \mathrm{~Pa}, 70 \mathrm{~W}$, and $150 \mathrm{sec}$, an ENC array without gold NPs can be formed. After etching, the residual resists were completely cleaned by soaking the sample in an acetone solvent for $30 \mathrm{~min}$ and implementing an ultrasonic bath for $3 \mathrm{~min}$. The cleaned PIC nanostructures consist of pure gold without any residual resist, which was verified by energy dispersive X-ray spectroscopy characterization (refer to Section 1 of the Supplementary Information for details). The quality and geometry of the fabricated structures were examined by scanning electronic microscopy (SEM, FEI Serion 2000).

\section{RESULTS AND DISCUSSION}

The PIC array is designed to produce strong CFE at an expected SERS excitation wavelength of $720 \mathrm{~nm}$. As previously mentioned, strong Fano resonance may be generated in the PIC array via the coupling between the NP mode and the cavity mode of the nanovoid. Therefore, the array should be designed to achieve cavity resonance at a target wavelength of approximately $720 \mathrm{~nm}$. By numerical simulations (refer to the details in Section 2 of the Supplementary Information) and according to previous studies, ${ }^{26-30}$ we determined that although the cavity resonance modes are generated by individual cavities, their spectral positions can be effectively influenced by the lattice constant when the cavities are arranged in a periodic lattice. By numerical optimization and considering the fabrication capabilities, we select the array period $p=250 \mathrm{~nm}$, the nanocube width $l=150 \mathrm{~nm}$ (by which the coupling between the adjacent PIC units is prevented), the cavity diameter $d_{\mathrm{c}}=120 \mathrm{~nm}$, and the height of the truncated cavity $t=60 \mathrm{~nm}$. With these parameters, the far-field reflectance and transmittance spectra of the ENC array were simulated and shown in Figure 1c. The reflectance spectrum has two evident resonance peaks in the wavelength range of $700 \mathrm{~nm}$ to $1000 \mathrm{~nm}$, which are attributed to the ${ }^{1} \mathrm{D}_{+}$and ${ }^{1} \mathrm{P}_{+}$modes of the truncated nanocavity under normal incidence. ${ }^{28}$ This can be verified by the simulated field distributions in the ENC in Figure 3e and f. Note that our ENC array is situated on a silica substrate, which differs from the case of Cole et $a .^{28}$, in which the empty nanovoids are embedded in an infinitely thick gold substrate. This situation explains why the simulated field distributions of the two modes slightly differ from the simulated field distributions in Cole et al. ${ }^{28}$. However, the key features of the mode profiles remain identical, which indicates the excitation of the two modes. With these selected void parameters, the ${ }^{1} \mathrm{D}_{+}$mode is tuned to be very close to the target wavelength of $720 \mathrm{~nm}$. We are especially interested in this mode because the field hot spots of this mode (refer to Figure 3e) are primarily located at the bottom of the void to ensure strong coupling with the field of the gold NP.

The last important parameter to be determined for the PIC array is the size of the gold NP. As demonstrated by the simulated reflectance spectrum of a PIC array with $20 \mathrm{~nm}$ gold NPs (i.e., diameter $d_{\mathrm{p}}=$ $20 \mathrm{~nm}$ ) in Figure 1b, the main consequence of introducing a gold NP in each nanovoid is the generation of an additional peak between the ${ }^{1} \mathrm{D}_{+}$and ${ }^{1} \mathrm{P}_{+}$peaks. The peak positions of the ${ }^{1} \mathrm{D}_{+}$and ${ }^{1} \mathrm{P}_{+}$cavity modes also slightly shift due to the perturbation by the gold NP. This 

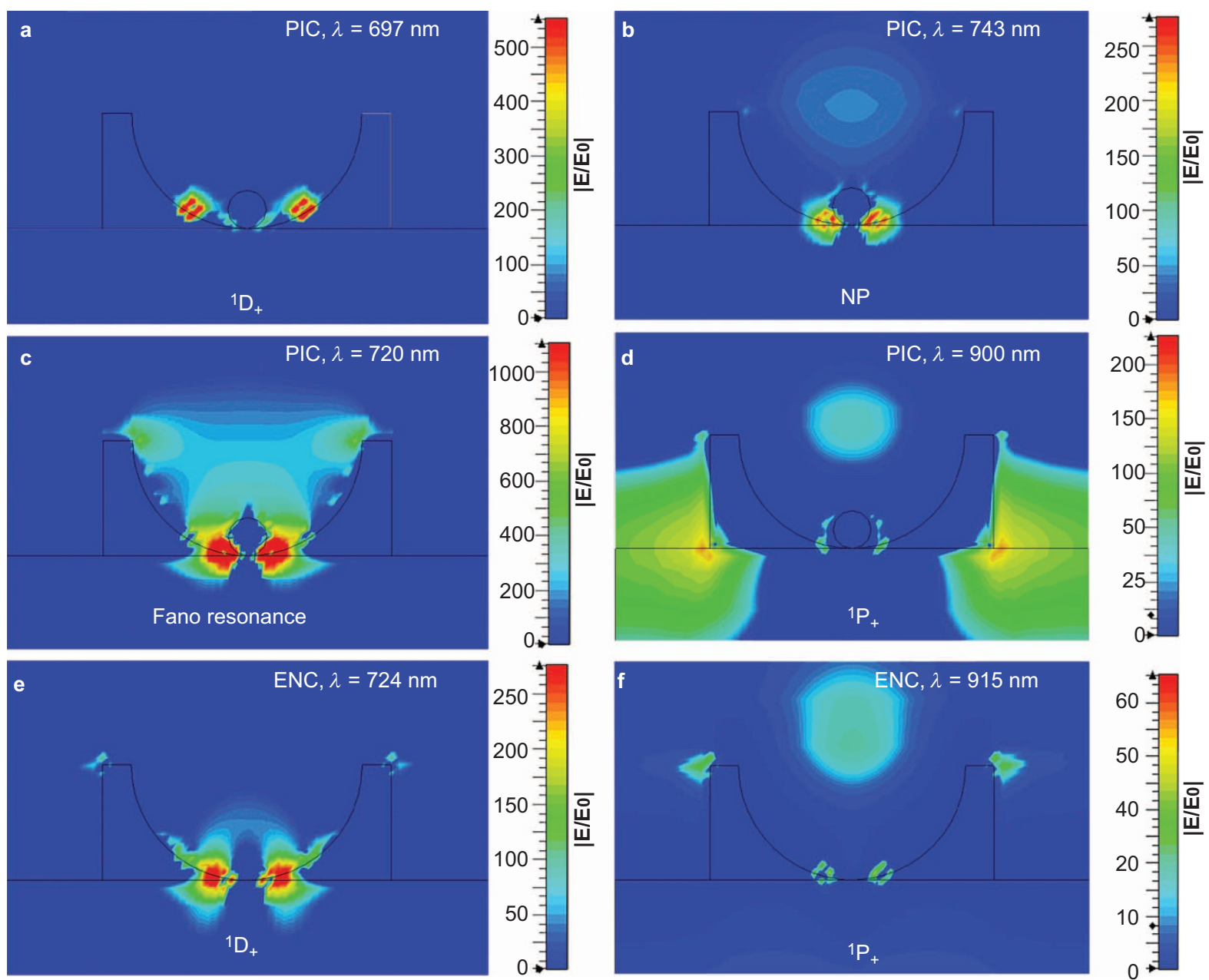

Figure 3 Simulated normalized electric field amplitude distributions in the PIC array and the ENC array. (a) The ${ }^{1} D_{+}$mode in the PIC array at wavelength 697 nm. (b) The NP mode in the PIC array at wavelength $743 \mathrm{~nm}$. (c) The Fano resonance in the PIC array at wavelength $720 \mathrm{~nm}$. (d) The ${ }^{1} \mathrm{P}+$ mode in the PIC array at wavelength $900 \mathrm{~nm}$. (e) The ${ }^{1} D_{+}$mode in the ENC array at wavelength $724 \mathrm{~nm}$. (f) The ${ }^{1} \mathrm{P}_{+}$mode in the ENC array at wavelength $915 \mathrm{~nm}$.

additional peak is caused by a 'NP mode' that is induced by the coupling between the gold NP and its image. ${ }^{30}$ Compared with the nanocavity modes ${ }^{1} \mathrm{D}_{+}$and ${ }^{1} \mathrm{P}_{+}$, which cause relatively broad resonances (the super-radiant bright modes), the NP mode is a subradiant dark mode and causes narrow resonance. When the NP mode is close to the ${ }^{1} \mathrm{D}_{+}$mode, they have strong interference that causes strong Fano resonance, which is manifest as an asymmetric resonance peak in the transmittance spectrum of the PIC array (refer to the red curve in Figure 1b) and does not exist in the ENC array (refer to the red curve in Figure 1c). We have also thoroughly investigated the influence of the NP size on the Fano resonance peak position and the maximum field enhancement factor at the target wavelength $720 \mathrm{~nm}$, as shown in Figure 1e and $\mathrm{f}$. With an increase in NP size, the Fano resonance peak develops a red shift (as indicated by the arrows in Figure 1f). When $d_{\mathrm{p}}$ $=20 \mathrm{~nm}$, the Fano peak is tuned to be exactly located at the target wavelength of $720 \mathrm{~nm}$ and the corresponding maximum field enhancement factor is also attained at this wavelength (as shown in Figure 1e). Thus, we select $d_{\mathrm{p}}=20 \mathrm{~nm}$ in our PIC array design. Note that when the NP size is increased, the NP mode peak may causes red shift, which results in weaker interference with the ${ }^{1} \mathrm{D}_{+}$mode but stronger interference with the ${ }^{1} \mathrm{P}_{+}$mode (as denoted by the pink curve in Figure 1f).
To verify the far-field responses of the PIC array, we have measured the transmittance of the fabricated PIC and ENC arrays by the spectroscopic ellipsometer VASE (J. A. Woollam Co.) in the wavelength range from $400 \mathrm{~nm}$ to $900 \mathrm{~nm}$ under normal incidence, as shown in Figure 1d. The experimental spectra are consistent with the theoretical predictions, which verifies the expected Fano resonance. The slight differences in the spectra are most likely attributed to the deviation in the structural parameters of the fabricated samples from theoretical designs.

As previously discussed, the ultimate goal of generating and tuning Fano resonance by mode hybridization in the plasmonic PIC system is to produce a strong CFE effect. We have simulated field distributions in the PIC array, as shown in Figure $3 a-d$. The ${ }^{1} D_{+}$mode (Figure 3a) and ${ }^{1} \mathrm{P}_{+}$mode (Figure $3 \mathrm{~d}$ ) retain their mode profile features (although perturbed somehow by the gold NP). The Fano interference of the ${ }^{1} \mathrm{D}_{+}$mode with the NP mode (Figure $3 \mathrm{~b}$ ) produces remarkable CFE at the target wavelength of $720 \mathrm{~nm}$ (as shown in Figure 3c), which result in significantly enhanced field hot spots and a slight change in the distribution of the hot spots in the cavity. These results are very important and beneficial for enhancing the light-matter interaction in the cavities. The maximum simulated field enhancement factor (i.e., the normalized field amplitude $\left|\mathbf{E} / \mathbf{E}_{0}\right|$, where $\mathbf{E}_{0}$ is the 
amplitude of the incident electric field) is $1.2 \times 10^{3}$, whereas the enhancement factor in the ENC array without the CFE effect is only $10^{2}$, which demonstrates that the CFE effect can significantly improve the field enhancement factor.

Note that we did not consider the finite contact area between the gold NP and the gold void in these simulations, that is, the contact between the NP and the void was assumed to be an ideal point. In the fabricated samples, the physical contact formed during the etching process must have a nonzero area. In Section 3 of the Supplementary Information, we examined the influence of the contact area on both the far-field and near-field responses of the PIC array. The findings indicate that an increase in the contact area may cause a slight decrease in the Fano resonance peak (although the resonance peak profile remains almost unchanged) and a decrease in the near-field enhancement. For example, when the radius of the contact area is assumed to be $4 \mathrm{~nm}$, the maximum near-field enhancement factor in the PIC decreases to approximately $4 \times 10^{2}$. Therefore, the calculated field enhancement factor of $1.2 \times 10^{3}$ is the most optimistic value. In practical samples, the field enhancement may be degraded due to the finite contact area and other fabrication defects.

To corroborate the field enhancement in the PIC array, we have fabricated some samples of ENC and PIC arrays, as displayed in the SEM images in Figure $2 b$ and $c$, respectively. The insets show enlarged top-view images of the samples. As shown in Figure 2c, only one gold NP is situated in each nanocavity, which verifies the reliability and quality of the fabrication method. In the ENC array in Figure $2 b$, no gold NP is observed in the nanocavity.

As a demonstration of the SERS performance of the PIC array, we have conducted a comparative experiment using the PIC array and the ENC array as SERS substrates for probing 4-ATP molecules (SigmaAldrich Co., with bulk concentration $0.1 \mu \mathrm{M}$ ). The reference Raman signal was detected from an unpatterned area of the gold film. All Raman spectra removed the baseline and were fitted using a Gaussian-Lorentzian line shape. In the SERS experiment, 4-ATP molecules were dosed onto the sample substrates via the chemical bonds between the gold atoms and sulfur atoms on the surface when the substrates were soaked for three hours in the 4-ATP solution and washed several times with ethanol to obtain a monolayer of 4-ATP molecules on the samples.

The back-scattering Raman spectra were collected by a LabRam microRaman system (Jobin-Yvon/ISA HR). A super-continuum light source (Fianium, SC400-PP) was tuned to emit light at an excitation wavelength of $720 \mathrm{~nm}$ with an output power $0.6 \mathrm{~mW}$ and an integration time of $10 \mathrm{~s}$. A thermoelectrically cooled charge-coupled device (CCD) array detector and a BH-2 microscope with a $100 \times$ shortfocal-length objective lens with a 0.5 numerical aperture were employed. The diameter of the Raman signal probing area was approximately $1 \mu \mathrm{m}$. The SERS spectra were collected in the Raman shift range of $400 \mathrm{~cm}^{-1}$ to $1800 \mathrm{~cm}^{-1}$.

Figure 4 shows the measured SERS spectra of the 4-ATP molecules on the PIC array, the ENC array, and the reference gold film. Note that the reference spectrum is multiplied by a factor of 10 . The SERS signal collected from the PIC array, which is approximately four times stronger than the SERS signal collected from the ENC array, is the strongest signal. The SERS enhancement factor $(E F)$ can be estimated by ${ }^{6}$

$$
E F=\frac{I_{\text {SERS }} / N_{\text {surf }}}{I_{\text {bulk }} / N_{\text {bulk }}}
$$

where $I_{\text {SERS }}$ and $I_{\text {bulk }}$ are the Raman signal intensities at the characteristic vibration mode at $1078 \mathrm{~cm}^{-1}$ of 4 -ATP molecules doped on the

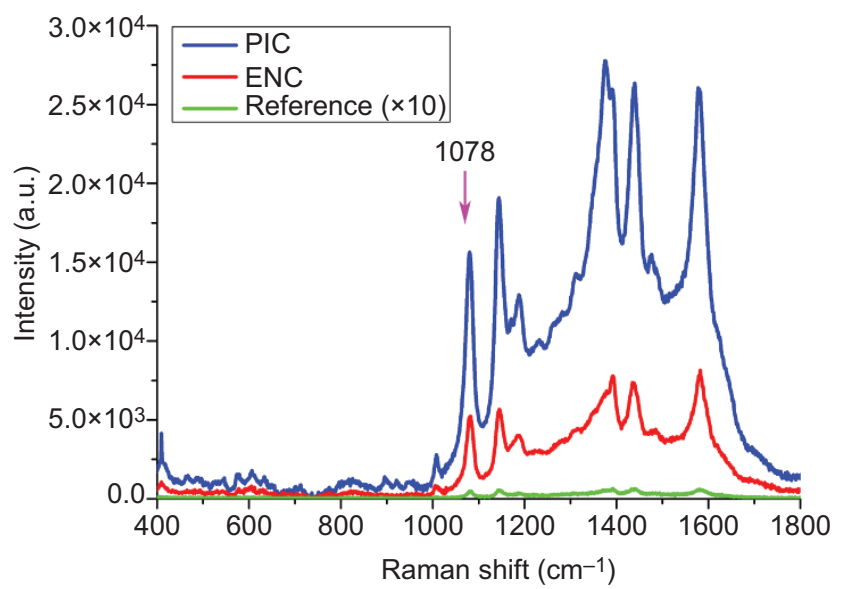

Figure 4 Experimentally measured Raman spectra of the 4-ATP molecules on different substrates: a PIC array, an ENC array, and an unpatterned gold film for reference. The $1078 \mathrm{~cm}^{-1}$ band is the fingerprint vibration wavenumber of the 4-ATP molecules.

nanostructured samples and on the surface of the reference flat gold film, respectively, and $N_{\text {surf }}$ and $N_{\text {bulk }}$ are the estimated number of 4ATP molecules doped on the sample and number of bulk 4-ATP molecules in the probing light spot area, respectively. Details on the estimation of $N_{\text {bulk }}$ and $N_{\text {surf }}$ are given in Section 4 of the Supplementary Information. According to Equation (1), the experimental SERS enhancement factor of the PIC array is estimated to be $2 \times 10^{7}$, which is a relatively conservative estimate. Because the collected Raman signal from the PIC array is primarily derived from the gold NP with strong field hot spots, the real number of 4-ATP molecules $N_{\text {surf }}$ adsorbed on the NP may be significantly smaller, which may produce a larger estimated experimental SERS enhancement factor (maximum of $10^{9}$ ).

The SERS experiment provides persuasive evidence that the PIC array has significantly improved SERS performance due to the CFE effect boosted by Fano resonance in the PIC nanoantennas. Therefore, the PIC array has great potential for use as a highly active SERS substrate.

\section{CONCLUSIONS}

We have proposed a plasmonic PIC array that consists of nanoantennas, in which a $20 \mathrm{~nm}$ gold NP is placed at the bottom of a $120 \mathrm{~nm}$ truncated hemispherical gold nanovoid. Using plasmonic mode hybridization in this multiscale system, strong Fano resonance can be generated due to the simultaneous excitation and intercoupling of the NP mode with a high-order cavity mode, which significantly boost the CFE in the PIC antenna. The maximum enhancement factor of the localized field amplitude ranges from $10^{2}$ to $10^{3}$, which is significantly higher than the maximum enhancement factor in a reference ENC array. To realize the PIC array with high performance, we have developed a cost-effective, efficient and reliable nanofabrication method that involves RT-NIL and anisotropic RIE. Using this technique, only one gold NP is located in each nanovoid and the NP size can be finely controlled to be approximately $20 \mathrm{~nm}$, which are crucial for realizing a significant CFE effect in the PIC array. As a demonstration of the CFE performance of the PIC array, the PIC samples were fabricated and employed as SERS substrates for detecting 4-ATP molecules. The achieved experimental SERS enhancement 
factor is $2 \times 10^{7}$, which demonstrates the strong field enhancement in the PIC array and its great potential for applications.

\section{ACKNOWLEDGMENTS}

We acknowledge the support by the National Natural Science Foundation of China (Projects No. 11474180, and No. 61227014) and the Ministry of Science and Technology of China (Project No. 2011BAK15B03).

1 Prodan E, Radloff C, Halas NJ, Nordlander P. A hybridization model for the plasmon response of complex nanostructures. Science 2003; 302: 419-422.

2 Stockman MI, Faleev SV, Bergman DJ. Coherent control of femtosecond energy localization in nanosystems. Phys Rev Lett 2002; 88: 067402.

3 Li K, Stockman MI, Bergman DJ. Self-similar chain of metal nanospheres as an efficient nanolens. Phys Rev Lett 2003; 91: 227402.

4 Ameling R, Giessen H. Microcavity plasmonics: strong coupling of photonic cavities and plasmons. Laser Photon Rev 2013; 7: 141-169.

5 Lukyanchuk B, Zheludev N, Maier SA, Halas NJ, Nordlander P et al. The Fano resonance in plasmonic nanostructures and Metamaterials. Nat Mater 2010; 9. 707-715.

6 Ru EL, Etchegoin P. Principle of Surface Enhanced Raman Spectroscopy. Oxford: Elsevier; 2009.

7 Kneipp K, Wang Y, Kneipp H, Perelman LT, Itzkan I et al. Single molecule detection using Surface-Enhanced Raman Scattering (SERS). Phys Rev Lett 1997; 78: 1667.

8 Chirumamilla M, Toma A, Gopalakrishnan A, Das G, Zaccaria RP et al. 3D nanostar dimers with a sub-10-nm gap for single-/few-molecule surface-enhanced Raman scattering. Adv Mater 2014; 26: 2353-2358.

9 Zhang R, Zhang Y, Dong ZC, Jiang S, Zhang $C$ et al. Chemical mapping of a single molecule by plasmon-enhanced Raman scattering. Nature 2013; 498: 82-86.

10 Fan JA, Bao K, Wu C, Bao J, Bardhan R et al. Fano-like interference in self-assembled plasmonic quadrumer clusters. Nano Lett 2010; 10: 4680-4685.

11 Toroghi S, Kik PG. Cascaded field enhancement in plasmon resonant dimer nanoantennas compatible with two-dimensional nanofabrication methods. App Phys Lett 2012; 101: 013116.

12 Çetin AE, Artar A, Turkmen M, Yanik AA, Altug H. Plasmon induced transparency in cascaded $\pi$-shaped metamaterials. Opt Express 2011; 19: 22607-22618.

13 Bidault S, García de Abajo SF, Polman JA. Plasmon-based nanolenses assembled on a well-defined DNA template. J Am Chem Soc 2008; 130: 2750-2751.

14 Kravets VG, Zoriniants G, Burrows CP, Schedin F, Casiraghi C et al. Cascaded optical field enhancement in composite plasmonic nanostructures. Phys Rev Lett 2010; 105 246806.

15 Kravets VG, Zoriniants G, Burrows CP, Schedin F, Geim AK et al. Composite Au nanostructures for fluorescence studies in visible light. Nano Lett 2010; 10: 874 879.

16 Höppener C, Lapin ZJ, Bharadwaj P, Novotny L. Self-similar gold nanoparticle antennas for a cascaded enhancement of the optical field. Phys Rev Lett 2012; 109: 017402.

17 Li X, Tan Q, Bai B, Jin G. Non-spectroscopic refractometric nanosensor based on a tilted slit-groove plasmonic interferometer. Opt Express 2011; 19: 20691-20703.

18 Kumar K, Duan H, Hegde RS, Koh SCW, Wei JN et al. Printing colour at the optical diffraction limit. Nat Nanotechn 2012; 7: 557-561.

19 Zhu Z, Bai B, Duan H, Zhang H, Zhang M et al. M-shaped grating by nanoimprinting: a replicabe, large-area, highly active plasmonic surface-enhancement Raman scattering substrate with nanogaps. Small 2014; 10: 1603-1611.

20 Zhang S, Genov DA, Wang Y, Liu M, Zhang X. Plasmon-induced transparency in metamaterials. Phys Rev Lett 2008; 101: 047401.
21 Hao F, Sonnefraud Y, Dorpe PV, Maier SA, Halas NJ et al. Symmetry breaking in plasmonic nanocavities: subradiant LSPR sensing and a tunable Fano resonance. Nano Lett 2008; 8: 3983-3988.

22 Fano U. Effects of configuration interaction on intensities and phase shifts. Phys Rev $1961 ; 124: 1866-1878$

23 Sonnefraud Y, Verellen N, Sobhani H, Vandenbosch GAE, Moshchalkov VV et al. Experimental realization of subradiant, superradiant, and Fano resonances in ring/ disk plasmonic nanocavities. ACS Nano 2010; 4: 1664-1670.

24 Radloff C, Halas NJ. Plasmonic properties of concentric nanoshells. Nano Lett 2004; 4: 1323-1327.

25 Dregely D, Hentschel M, Giessen H. Excitation and tuning of higher-order Fano resonances in plasmonic oligomer clusters. ACS Nano 2011; 5: 8202-8211.

26 Sugawara Y, Kelf TA, Baumberg JJ, Abdelsalam ME, Bartlett PN. Strong coupling between localized plasmons and organic excitons in metal nanovoids. Phys Rev Lett 2006; 97: 266808.

27 Kelf TA, Sugawara Y, Cole RM, Baumberg JJ, Abdelsalam ME et al. Localized and delocalized plasmons in metallic nanovoids. Phys Rev B 2006; 74: 245415.

28 Cole RM, Baumberg JJ, de Abajo FJG, Mahajan S, Abdelsalam M et al. Understanding plasmons in nanoscale voids. Nano Lett 2007; 7: 2094-2100.

29 de Abajo FJG. Light scattering by particle and hole arrays. Rev Mod Phys 2007; 79. 1267-1290.

30 Huang FM, Wilding D, Speed JD, Russell AE, Bartlett PN et al. Dressing plasmons in particle-in-cavity architectures. Nano Lett 2011; 11: 1221-1226.

31 Hao F, Nehl CL, Hafner JH, Nordlander P. Plasmon resonances of a gold nanostar. Nano Lett 2007; 7: 729-732.

32 Deng X, Braun GB, Liu S, Sciortino PF, Koefer B et al. Single-order, subwavelength resonant nanograting as a uniformly hot substrate for surface-enhanced Raman spectroscopy. Nano Lett 2010; 10: 1780-1786.

33 Miroshnichenko AE, Flach S, Kivshar YS. Fano resonances in nanoscale structures. Rev Mod Phys 2010; 82: 2257-2298.

34 Hartmanni MJ, Brandao FGSL, Plenio MB. Strongly interacting polaritons in coupled arrays of cavities. Nat Phys 2006; 2 : 849-855.

35 Ye J, Lagae L, Maes G, Dorpe PV. A versatile method to fabricate particle-in-cavity plasmonic nanostructures. J Mater Chem 2011; 21: 14394-14397.

36 Chen B, Meng G, Zhou F, Huang Q, Zhu C et al. Ordered arrays of Au-nanobowls loaded with Ag-nanoparticles as effective SERS substrates for rapid detection of PCBs. Nanotechn 2014; 25: 145605

37 Li X, Zhang Y, Shen ZX, Fan HJ. Highly ordered arrays of particle-in-bowl plasmonic nanostructures for surface-enhanced Raman scattering. Small 2012; 8: 2548-2554.

38 Chandra M, Dowgiallo A-M, Knappenberger KL. Magnetic dipolar interactions in solid gold nanosphere dimers. J Am Chem Soc 2012; 134: 4477-4480.

39 Shegai T, Li Z, Dadosh T, Zhang Z, Xu H et al. Managing light polarization via plasmonmolecule interactions within an asymmetric metal nanoparticle trimer. PNAS 2008; 105: 16448-16453.

40 Ou FS, Hu M, Naumov I, Kim A, Wu W et al. Hot-spot engineering in polygonal nanofinger assemblies for surface enhanced Raman spectroscopy. Nano Lett 2011; 11: 2538-2542.

41 CST Microwave Studio, www.cst.com.

42 Johnson PB, Christy RW. Optical constants of the noble metals. Phys Rev B 1972; 6 : 4370.

43 Mansky P, Liu Y, Huang E, Russell TP, Hawker C. Controlling polymer-surface interactions with random copolymer brushes. Science 1997; 275: 1458-1460.

\footnotetext{
(c) (i) This work is licensed under a Creative Commons Attribution 3.0 Unported License. The images or other third party material in this article are included in the article's Creative Commons license, unless indicated otherwise in the credit line; if the material is not included under the Creative Commons license, users will need to obtain permission from the license holder to reproduce the material. To view a copy of this license, visit http://creativecommons.org/licenses/by/3.0/
} 